\title{
Machine Learning Approach for Crop Selection based on Agro-Climatic Conditions
}

\author{
Prof.K.D.Yesugade ${ }^{1}$,Aditi Kharde ${ }^{2}$, Ketki Mirashi ${ }^{3}$, Kajal Muley ${ }^{4}$ Hetanshi Chudasama $^{5}$ \\ Assistant Professor, Dept. of computer Engineering, BVCOEW, Pune, India ${ }^{1}$ \\ Students, Dept. of computer Engineering, BVCOEW, Pune, India ${ }^{2,3,4,5}$
}

\begin{abstract}
Farming helps to meet the basic needs of human and their civilization by providing food, clothing, shelters, medicine and recreation. Hence, agriculture is the most important enterprise in the globe. Agriculture provides food, feed, fibre, fuel, furniture, raw materials and materials for and from factories; provides a free fare and fresh environs, abundant food for driving out dearth; favours friendship by eliminating battle. One of the reasons for the shortage of food across the country can be choice of unsuitable crop for cultivation. The proposed project will contain information of different crops and will suggest the granger crop which is suitable for cultivation based on the geographic area and climatic status such as temperature, moisture and humidity by making use of different sensors.
\end{abstract}

Keywords: Climate, Sensors, climate, agricultural productivity, crop production, prediction, Machine Learning

\section{INTRODUCTION}

Agriculture helps to meet the basic needs of human being and their civilization by providing nutrient, clothing, shelters, medicine and recreation. Hence, agriculture is the most important enterprise in the world. It is a productive whole where the free gifts of nature namely land, light, air, temperature and rain water etc., are integrated into single primary unit indispensable for human beings. Secondary productive units namely animals including livestock, birds and insects, feed on these primary building block and provide concentrated products such as meat, milk, wool, eggs, honey, silk and lac. Therefore the term agriculture means cultivation of land. i.e.,the science and art of producing crops and livestock for economic purposes. It is also referred as the science of producing crops and livestock from the natural resources of the earth. The primary aim of agriculture is to cause the land to produce more abundantly, and at the same time , to protect it from deterioration and misuse. It is synonymous with farming-the production of food, fodder and other industrial materials. India is the largest producer and consumer of crops in the world, constituting 75\% of world production and consuming $90 \%$ of the world production. Other major countries are Myanmar, Kenya, Uganda and Malawi. Crop accounts for about 20 percent of the total pulse production of the country . India annually imports $2-3$ lakh tones of which $95 \%$ is from Myanmar. India annually produces about 2.0-2.5 million tonnes and the production has been stagnant in the past 10 years. The shift in cultivation from pulses to commercial crops and lack of technological innovations to increase yields has hindered the rise in output. The major producing states are Maharashtra , Uttar Pradesh, Orissa and Karnataka. Among these, Maharashtra is largest producer of crops which constitutes about $34 \%$ and these four states contribute nearly $70 \%$ of total output in the country.

\section{LITERATURE SURVEY}

\section{Agricultural Production Output Prediction Using Supervised Machine Learning Techniques}

\section{Md. TahmidShakoor, KarishmaRahman, SumaiyaNasrinRayta, AmitabhaChakrabarty}

This paper gives the information about the crop that should be selected forrearingso as to maximize the profit. The paper includes the research of six major crops including three rice species like Aus, Aman ,Boro and other crops like Potato, Wheat and Jute .This paper nidus on use of supervised machine learning techniques.The dataset is taken from Bangladesh Agricultural Research council according to area. The algorithms used in the paper are decision tree learning -ID3 and K Nearest Neighbour_algorithm[1]

2. Machine learning approach for forecasting crop yield based on climatic parameters . S.Veenadhari, Dr. Bharat Misra, Dr.CD Singh

In the present study authors have implemented a web application which is named as "Crop Advisor ". It gives prediction about the climatic conditions which affect the crop yield. In this paper to discover the effective climatic terms that affect the crop yield in Madhya Pradesh,C4.5 algorithm is used. The other parameters such as agro-input parameters are not considered in this application as they differ with change in space and measure of individual fields.[2] 


\section{Crop Selection Method Based on Various Environmental Factors Using Machine Learning.} Nishit Jain, Amit Kumar, SahilGarud, Vishal Pradhan, PrajaktaKulkarni.

This paper assist user the method that would help them to choose the crop which will maximize the crawtakings by taking into retainer all the parameter which affect the growth of crop. The different parameters like environmental ,economical and other parameters related to the yield in nature can be analysed for prediction of accurate resultant role. The economical parameters includes demand for crop, market rate etc whereas environmental parameters includes quantity of rainfall, temperature, and type of soil. So all these factors are considered while predicting the most efficient crop to be cultivated based on season.[3]

\section{A Scalable Machine Learning System for Pre-Season Agriculture Yield Forecast}

Igor Oliveira, Renato L. F. Cunha, Bruno Silva, Marco A. S. Netto.

Yield prognosis is essential to department of agriculture stakeholders and can be obtained with the use of machine learning modeling and information coming from multiple sources. Most solutions for yield forecast rely on NDVI (Normalized Difference Vegetation Index) information,which is time-consuming to beacquired and processed. This system provides significantly useful results by the exempting the need for high-resolution remote control-preception data and allowing farmer to prepare for adverse climate influence on the harvest oscillation. In our studies, we forecast the soybean and maize takings for Brazil and America, which corresponded to $44 \%$ of the world's food production in 2016. Results show the error metric unit for soybean and maize yield forecasts are comparable to similar scheme that only provide yield forecast information in the first weeks to calendar month of the crop cycle.[4]

\section{Prediction of crop yield using machine learning}

\section{RushikaGhadge, JuileeKulkarni, Pooja More, Sachee Nene, Priya R L}

Based on the previous research, it is observed that there is an increased in suicide rate of farmers in Maharashtra over the years. There are many grounds behind it. The main cause of this is the farmers are unaware about the fertility of territory and lack of knowledge about the type of soil required for growing particular craw. Therefore the proposed system in this paper helps them to know about the quality of the soil and suggest them the crop that will maximize the return.

\section{Rice crop yield prediction in India using SVM (Support Vector Machine).}

NiketaGandhi,LeisaJ.Armstrong, OwaizPetkar, Amiya Kumar Tripathi

This paper includes dataset of 27 districts in Maharashtra. The techniques presented by this paper is SMO classifier using WEKA putzs. The dataset includes the parametric quantity such as precipitation, minimum temperature , maximum temperature, average temperature, production and yield for kharif season. The outcome of this study showed that the performance of SMO classifier is poor. It suggests to use other different such as Naive Bayes, BayesNetetc to get the more accurate results.

\section{PROPOSE SYSTEM}

i. In proposed system, we are using three sensors which will sensetemperature, humidity, moisture values of the field.

ii. The controller will receive weather parameters from sensors. Data collected by controller will get uploaded on cloud through Wi-Fi.

iii. Admin will store trained dataset on cloud.

iv. Values collected from hardware get compared with trained dataset.

v. After comparison crop suggestion will display crops according to weather parameters.

vi. Then user will select crop from suggestions to get its information like pesticide, fertilizer to be used, harvesting time, amount of water required etc.

vii. After selecting any crop from suggestion user can see information of crops which can be plant.

\section{IV.SYSTEM ARCHITECTURE}

The proposed system architecture mainly consists of three modules -hardware, cloud and an android application. The hardware module consists of three sensors temperature, moisture and humidity. The data fetched from sensors will get stored on the cloud using Wi-Fi chip ESP8266. The algorithm will work on fetched data and the results will be displayed to user on android application. 


\section{International Journal of Advanced Research in Computer and Communication Engineering}

Vol. 7, Issue 10, October 2018

Following diagram is proposed system's architecture diagram:

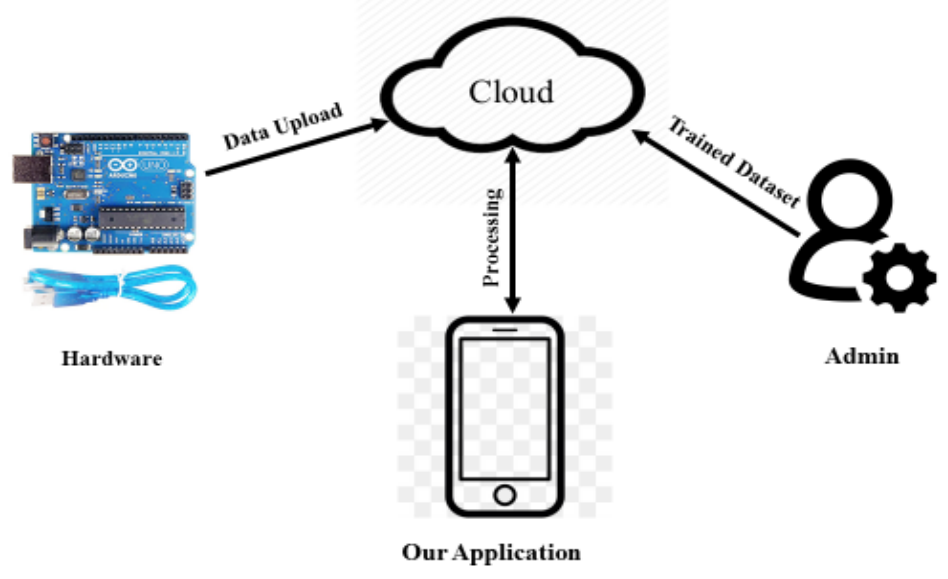

Figure 1: system architecture

\section{METHODOLOGIES}

Temperature Sensor: A temperature sensing device, that provides for temperature measurement through an electrical signal. The LM35 is one kind of commonly used temperature sensing element that can be used to measure temperature with an electrical $\mathrm{o} / \mathrm{p}$ comparative to the temperature (in ${ }^{\circ} \mathrm{C}$ ). It can measure temperature more correctly compare with a thermistor. This sensor generates a high output voltage than thermocouples and may not need that the output voltage is amplified. The LM35 has an output voltage that is proportional to the Celsius temperature. The scale factor is $.01 \mathrm{~V} /{ }^{\circ} \mathrm{C}$.

Humidity Sensor: Humidity sensor is working voltage $3.3 \mathrm{~V}-5$ V Humidity measurement ranges from 20 percent to 95 percent, humidity measurement error +-5 percent. Temperature measurement range $0-50$, measurement error +-2 degrees.DHT11 digital temperature and humidity sensor module is a composite Sensor contains a calibrated digital signal output of the temperature and humidity.

ESP8266: The ESP8266 is a low-cost Wi-Fi microchip with full TCP/IP and microcontroller capability produced by Shanghai-based Chinese manufacturer. The chip first came to the attention of western makers in August 2014 with the ESP-01 module, made by a third-party manufacturer Ai-Thinker. This small module allows microcontrollers to connect to a Wi-Fi network and make simple TCP/IP connections using Hayes-style commands.

Arduino: Arduino is an open-source electronics platform based on easy-to-use hardware and software program. Arduino boards are able to read inputs - light on a sensor and turn it into an output - activating a motor, turning on an LED, publishing something online.

K-Means Clustering: Clustering is used to group the items with same properties . K means is one of the most widely used algorithm of clustering. . K means clusters all items into different clusters depending on nearest mean value .K means uses centroid method as well as Euclidean distance method to group the items. Here in the dataset we have three major parameters namely Temperature, Moisture and Humidity. K means algorithm will form the clusters of the observations available in the dataset based on the temperature, moisture and humidity range. Depending on these clusters, the crops family with matching environmental conditions are determined.

\section{ADVANTAGES}

- Maximize the crop yield.

- The proposed system is helpful in identifying the crops suitable for cultivation in the given environmental conditions

- The information related to life cycle of selected crop is provided.

\section{APPLICATION}

- $\quad$ The proposed system can be used by farmers individually.

- It is can also be used in soil investigating centres. 


\title{
IJARCCE
}

\section{International Journal of Advanced Research in Computer and Communication Engineering}

\author{
Vol. 7, Issue 10, October 2018
}

\section{CONCLUSION}

Since the yield of farm highly depend on the crop selected for cultivation and environmental parameters therefore proper selection of crop before cultivation is important in farming. This system can be a great help in deciding the proper crop as per the given climatic conditions which will help to maximize yield rate.

\section{REFERRENCES}

[1]. Md. TahmidShakoor, KarishmaRahman, SumaiyaNasrinRayta, AmitabhaChakrabarty, "Agricultural Production Output Prediction Using Supervised Machine Learning Techniques", IEEE, 2017

[2]. S.Veenadhari, Dr. Bharat Misra, Dr.CD Singh, "Machine learning approach for forecasting crop yield based on climatic parameters", International Conference on Computer Communication and Informatics (ICCCI -2014), Jan. 03 -05, 2014.

[3]. Nishit Jain, Amit Kumar, SahilGarud, Vishal Pradhan, PrajaktaKulkarni, "Crop Selection Method Based on Various Environmental Factors Using Machine Learning”, International Research Journal of Engineering and Technology (IRJET), Volume: 04 Issue: 02 , February -2017.

[4]. Igor Oliveira, Renato L. F. Cunha, Bruno Silva, Marco A. S. Netto, "A Scalable Machine Learning System for Pre-Season Agriculture Yield Forecast", 14th IEEE eScience, https://arxiv.org/abs/1806.09244

[5]. R. NageswaraRao, B.Sridhar," Iot Based Smart Crop-Field Monitoring and Automation Irrigation System ", IEEE Second International Conference on Inventive Systems and Control (ICISC), 2018.

[6]. EkataGhadage, VibhavariKharate, Parnika Mane, SamruddhiPimpale," Smart Irrigation and Crop Planning System:usingArduino Microcontroller", International Journal of Advanced Research in Computer and Communication Engineering, Vol. 6, Issue 1, January 2017,DOI 10.17148/IJARCCE.2017.6186.

[7]. Prof.K.D.Yesugade,SonalBathiya,Priya Bora,Nikita Waykule,’Agro-sense:A Mobile App For Efficient Farming System Using Sensors”, International Research Journal of Engineering and Technology (IRJET), Volume: 04 Issue: 04,,April -2015. 